\title{
Prüifungsstress meistern
}

Caroline Lutz-Kopp, Maria Gropalis

Prüfungen zu meistern ist nicht nur eine Frage der fachlichen Kompetenz. Starke Prüfungsängste können einen daran hindern, das eigene Potenzial voll auszuschöpfen und sich beruflich (weiter) zu qualifizieren. Die Bewältigung von Prüfungsängsten kann gelernt werden - manchmal helfen schon kleine Veränderungen in der Prüfungsvorbereitung oder während der Prüfung. Die Autorinnen geben zunächst einen Überblick über die Symptome, Entstehung und Aufrechterhaltung von Prüfungsängsten. Anschließend stellen sie konkrete und leicht umsetzbare Bewältigungsstrategien vor.

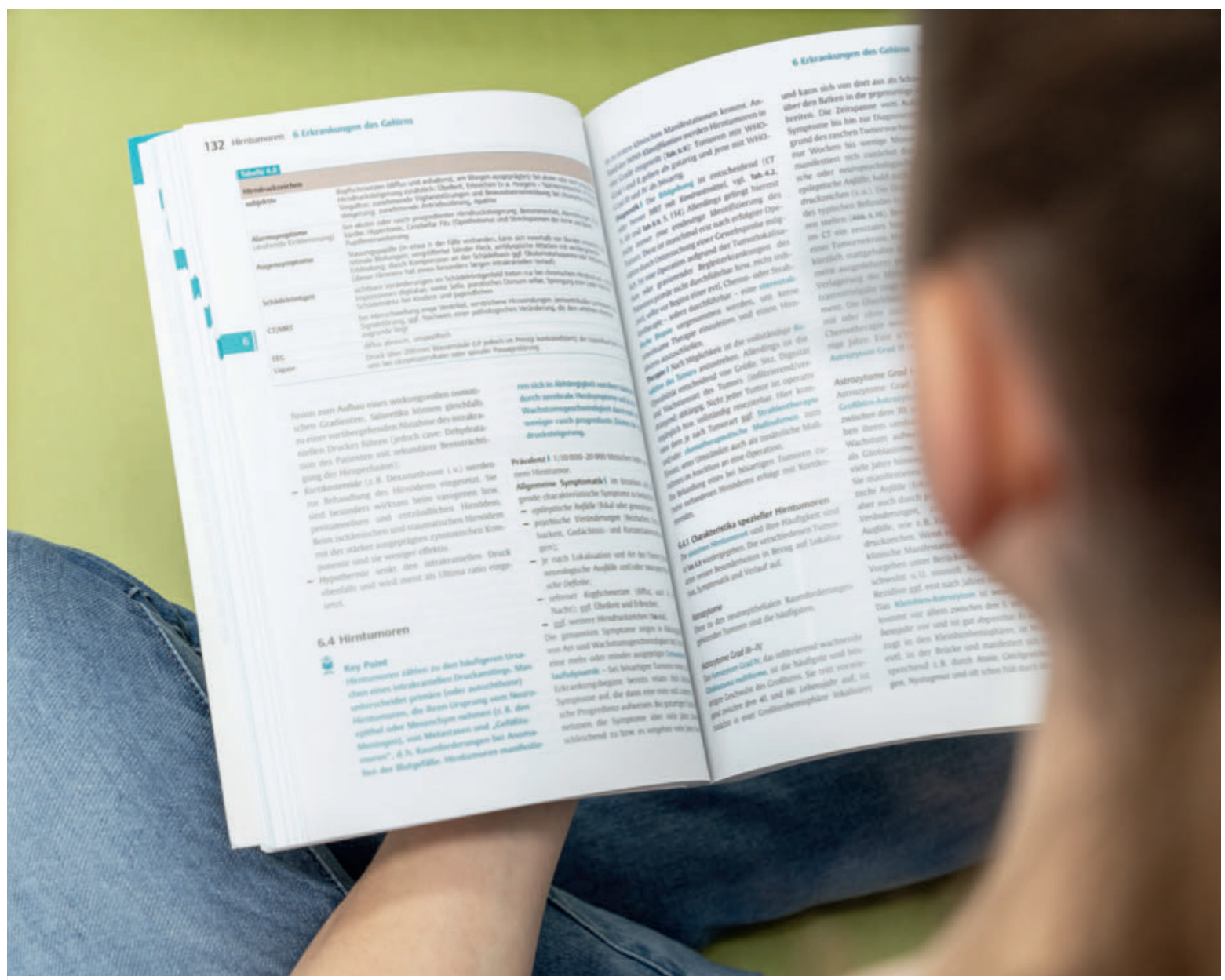

Wer die Prüfung als Herausforderung akzeptiert und Strategien gegen Prüfungsangst regelmäßig übt, hat gute Chancen, die Situation erfolgreich zu meistern. (Symbolbild/Quelle: Kirsten Oborny/Thieme Gruppe)

CNE (Certified Nursing Education) ist das multimediale Fortbildungsangebot von Thieme. Es wurde in enger Zusammenarbeit mit dem Deutschen Pflegerat e.V. (DPR) entwickelt.

Weitere Informationen finden Sie unter cne.thieme.de 
Vor Prüfungen aufgeregt zu sein, leichtes Herzklopfen zu haben oder die Sorge zu versagen - das kennen viele Menschen. Leichte Aufregung beziehungsweise Angst gehört zu Prüfungen dazu. Sie zeigt, dass einem die Prüfung wichtig ist und ist in der Regel der besonderen Leistungssituation angemessen. Denn Prüfungen stellen häufig den Beginn eines neuen Lebensabschnitts dar. Angst aktiviert den Körper und das Gehirn und stellt Energie für die Vorbereitung auf die Prüfung sowie Motivation zum Lernen bereit. Auch in der Prüfung selbst führt eine leichte Aktivierung dazu, dass der Prüfling wach und konzentriert ist. Ein mittleres Maß an Aufgeregtheit ist daher sinnvoll.

\section{Prüfungsängste und die Begleitsymptome}

Tritt die Angst allerdings so stark ausgeprägt und langanhaltend auf, dass die oder der Betroffene gar nicht mehr lernen kann, Prüfungen verschiebt, sodass sich zum Beispiel die Ausbildungsdauer verlängert, oder auch die allgemeine Lebensqualität dadurch eingeschränkt ist, handelt es sich um eine klinisch relevante, behandlungsbedürftige Prüfungsangst. Im ICD-10 wird diese unter den spezifischen Phobien diagnostiziert (ICD-10: F40.2) [1].

Herzrasen, Schwitzen, Zittern, Schlafprobleme und Konzentrationsstörungen sind einige der Symptome, die typischerweise mit Prüfungsängsten einhergehen. Angst kann verschiedene Aspekte einer Prüfung betreffen: die Vorbereitung, die Prüfung selbst oder das Ergebnis. Es kann auch einen Unterschied machen, ob es sich um eine mündliche oder schriftliche Prüfung handelt. Um Prüfungsangst als eine psychische Störung einzuordnen, dürfen die Ängste den Bedingungen der Prüfungsvorbereitung und der Prüfung selbst nicht angemessen sein. Hat eine Person zum Beispiel starke Angst, eine Prüfung nicht zu bestehen, da sie sich (tatsächlich) nicht ausreichend vorbereitet hat, handelt es sich nicht um Prüfungsangst, sondern um eine der Situation entsprechend angemessene Angst.

Prüfungsängste treten vor allem in der Schul- und Ausbildungszeit oder im Studium auf. Aber auch die Prüfung am Ende einer Zusatzqualifikation oder ein Bewerbungsgespräch können Prüfungsängste auslösen. Es handelt sich um eine Versagensangst in Leistungssituationen.

Zur Häufigkeit gibt es verschiedene Ergebnisse, bei unterschiedlichen Stichproben. Ein systematisches Review von Shapiro [2] ergab, dass 30\% der Krankenpflegeschülerinnen und -schüler von Prüfungsangst betroffen sind. Quek und Kollegen [3] kommen in einer Metaanalyse zu dem Ergebnis, dass 33,8\% der Medizinstudierenden an Prüfungsangst leiden. In der Psychotherapeutischen Beratungsstelle der Johannes Gutenberg-Universität Mainz gaben im Jahr $201820 \%$ der Ratsuchenden an, dass sie aufgrund
KRITERIEN FÜR EINE SPEZIFISCHE (ISOLIERTE) PHOBIE NACH ICD-10 (F40.2) [1]

A. Deutliche Furcht vor oder Vermeidung eines bestimmten Objektes oder einer bestimmten Situation.

B. Symptome autonomer Erregung (mindestens 2 der Symptome aus 1-14, darunter mindestens 1 Symptom aus $1-4$ ):

1. Herzklopfen

2. Schweißausbrüche

3. Tremor

4. Mundtrockenheit

5. Atembeschwerden

6. Beklemmungsgefühl

7. Schmerzen oder Missempfindungen in der Brust

8. Übelkeit

9. Schwindel

10. Derealisation oder Depersonalisation

11. Furcht vor Kontrollverlust

12. Angst zu sterben

13. Hitzewallungen

14. Gefühllosigkeit/Kribbelgefühle

C. Deutliche emotionale Belastung durch die Angstsymptome oder das Vermeidungsverhalten, und Einsicht, dass diese übertrieben sind.

D. Symptome beschränken sich auf die gefürchteten Situationen oder auf Gedanken daran.

von Prüfungsängsten eine psychologische Beratung aufsuchen [4].

\section{Die 4 Ebenen der Prüfungsangst}

Prüfungsängste äußern sich auf 4 Ebenen: emotional, physiologisch, gedanklich und im Verhalten [5]:

- Auf emotionaler Ebene zeigen sich starke Angst bis hin zu Panik. Begleitend können Niedergeschlagenheit, Hoffnungslosigkeit, Scham, Ärger und Versagensgefühle auftreten.

- Durch eine gesteigerte Ausschüttung des körpereigenen Hormons Adrenalin kommt es körperlich zu Herzklopfen, einem erhöhten Blutdruck, schnellerer Atmung und Schwitzen.

- Katastrophisierende Gedanken lösen das entsprechende Gefühl in der jeweiligen Situation aus. Typische negative Gedanken, die mit Prüfungsangst einhergehen, sind zum Beispiel: „Ich schaffe es nie, das alles zu lernen. Ich werde versagen. Ich bekomme in der Prüfung sicherlich kein Wort heraus und kann mich an nichts des Gelernten erinnern."

- Die Angst äußert sich weiterhin häufig in ungünstigen Verhaltensweisen während der Prüfungsvorbereitung, beispielweise Bewegungsmangel, „Frustessen“ sowie vermehrtem Konsum von Genuss- oder Rauschmitteln. Manchmal kommt es auch zur Vermeidung der angstauslösenden Situation, in dem Fall der Prüfung oder der Prüfungsvorbereitung. 


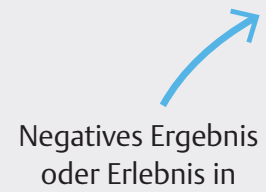

Negative Einschätzung

der eigenen Fähigkeiten

Bezug auf die Prüfung

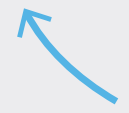

Erhöhte Anspannung

Teufelskreis der

Prüfungsangst

und/oder verminderte

Leistungsfähigkeit

Abb. 1 Teufelskreis der Prüfungsangst.

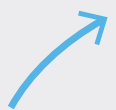

Ausweitung verhaltens

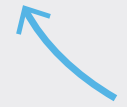

des Vermeidungs-
Unsicherheit

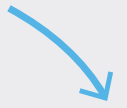

Vermeidung schwieriger Situationen (z. B. des Lernens oder der Prüfung)

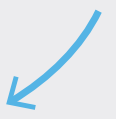

\section{Kurzfristige}

Erleichterung

(ich flüchte und habe deswegen momentan keine Angst) hin steigt die Anspannung, welche die Leistungsfähigkeit vermindert. Im schlimmsten Fall führt dies zu weiteren negativen Prüfungserlebnissen, was wiederum die Einschätzung der eigenen Fähigkeiten zusätzlich verschlechtert. Durch diesen Teufelskreis wird die Prüfungsangst aufrechterhalten ( $\triangleright$ Abb. 1).

Ein weiterer typischer aufrechterhaltender Kreislauf bei Prüfungsangst ist der Teufelskreis der Vermeidung. Unsicherheit und Ängste führen zur Vermeidung schwieriger Situationen. Vermeidung hat kurzfristig meist einen erleichternden Effekt, denn die unangenehme Situation (Lernen, Prüfung) wird nicht aufgesucht und die unangenehmen Gefühle (Angst, Unsicherheit), die damit einhergehen, werden vermieden. Langfristig jedoch kommt es dadurch zu weiterem Vermeidungsverhalten und zusätzlichen negativen Konsequenzen (zum Beispiel eine Prüfung wird nicht abgelegt und eine Zusatzqualifikation kann nicht beendet werden). Dies kann dann dazu führen, dass in immer mehr Situationen Unsicherheit und Angst empfunden werden ( $\triangleright$ Abb. 2).

\section{Prüfungsängste bewältigen}

Prüfungsangst kann individuell auf den 4 beschriebenen Ebenen (Gefühl, Körper, Gedanken und Verhalten) sehr unterschiedlich ausgeprägt sein. In der Bewältigung werden daher alle 4 Ebenen berücksichtigt. Die Wirksamkeit der im Folgenden beschriebenen kognitiv-verhaltenstherapeutisch orientierten Techniken wurde in zahlreichen Studien nachgewiesen [7][8].

Je nach Ausprägung der Prüfungsangst kann es hilfreich oder notwendig sein, sich bei der Bewältigung Unterstützung durch eine Psychologische Psychotherapeutin oder einen Psychologischen Psychotherapeuten zu suchen.

\section{Strategien auf körperlicher Ebene}

In einer Angstsituation reagiert der Körper mit einer Stressreaktion, indem er sich in Bereitschaft versetzt, zu kämpfen oder zu fliehen, um das Überleben zu sichern.

Besonders starke Erregung kann bis zu einem Blackout führen. Dabei handelt es sich um ein vorübergehendes funktionelles Gedächtnisproblem, das jeden treffen kann. Die Betroffenen können das Gelernte nicht erinnern und haben den Eindruck, einen „leeren Kopf“ zu haben. Häufig treten in Momenten des Blackouts zusätzlich angstverstärkende Gedanken, wie zum Beispiel „Jetzt ist alles vorbei“, auf. Strategien zum Umgang mit solchen Gedanken werden im nächsten Abschnitt erläutert.

Auf körperlicher Ebene geht es darum, allgemeine Entspannungskompetenzen aufzubauen, um die körperliche Erregung auf ein optimales Niveau zu senken [9]. Dies hilft nicht nur in Prüfungsphasen, sondern unterstützt dabei, das allgemeine Anspannungsniveau dauerhaft zu 
PRAXISTIPP: GEDANKEN VERÄNDERN

Zunächst geht es darum, sich die angstauslösenden Gedanken in Bezug auf die bevorstehende Prüfung bewusstzumachen. Dazu kann ein Gedankenprotokoll geführt werden [12]. Das hilft dabei, die Gedanken genauer zu analysieren und angstauslösende Gedanken zu identifizieren. In der 1.Spalte des Protokolls wird die konkrete Situation beschrieben. In der 2. werden alle Gedanken notiert, die einem in dem Moment durch den Kopf gehen. Und in die 3. Spalte werden die Konsequenzen dieser Gedanken auf die Gefühle und das Verhalten geschrieben ( Tab. 1).

Im nächsten Schritt werden dann in einer 4. Spalte hilfreichere und realistischere Gedanken für diese Situation gesucht und notiert. Die 5. Spalte dient einer Überprüfung dieser alternativen Gedanken. Hier werden die alternativen Konsequenzen auf die Gefühle und das Verhalten eingetragen. Das wirkt angstreduzierend und macht das Lernen leichter ( $\triangleright$ Tab. 2).

\section{Hilfreiche Fragen für die Entwicklung alternativer Gedanken}

Folgende Fragen helfen Betroffenen mit Prüfungsangst in der Einzelberatung, automatische Gedanken zu analysieren und zu hinterfragen sowie alternative Gedanken zu entwickeln:

- Hilft mir der Gedanke, das zu erreichen was ich möchte?

- Hilft er mir dabei, mich so zu verhalten, wie ich möchte?

- Wie fühle ich mich dabei?

- Trifft das, was ich denke, wirklich zu?

- Ist es schon bewiesen?

- Kann ich das überhaupt wissen?

- Was spricht dafür?

- Was spricht dagegen?

- Ist der Gedanke angemessen und realistisch?

- Wie wahrscheinlich ist das?

- Gibt es noch eine andere Sichtweise?

- Was würde ich einer Freundin/einem Freund raten?

- Was würde meine beste Freundin/mein bester Freund mir raten?

Denkmuster zu verändern ist ein langer Prozess. Daher wird den Klientinnen und Klienten in der Einzelberatung empfohlen, sich diese SpaltenProtokolle und vor allem die alternativen Gedanken in der Prüfungsvorbereitung regelmäßig durchzulesen.

senken und so stressigen Phasen resilienter zu begegnen. Wie bereits erwähnt, ist die Prüfungsleistung bei einem

\section{ÜBUNG: ATEM ZÄHLEN}

Eine schnelle und hilfreiche Technik, um Anspannung zu reduzieren, ist das „Atem zählen“. Die Übung kann jederzeit und überall angewendet werden und wird in einer aufrechten und bequemen Sitzhaltung durchgeführt.

In der Einzelberatung wird diese folgendermaßen angeleitet: Setzen Sie sich aufrecht und bequem hin und atmen Sie ein paar Mal tief ein und wieder aus. Dann beginnen Sie beim nächsten Einatmen zu zählen „eins“ und beim Ausatmen „zwei“. Wenn störende Gedanken kommen (und sie werden kommen), dann bemerken Sie diese, ohne sich zu ärgern, und kehren Sie mit Ihrer Aufmerksamkeit zu Ihrem Atem und dem Zählen zurück. Atmen und zählen Sie nun auf diese Weise bis etwa 20 beziehungsweise bis Sie eine Verminderung der Anspannung wahrnehmen können.

\section{EXKURS: DENKFEHLER}

Frühere belastende und ungünstige Erfahrungen sowie Lernprozesse führen zu kognitiven Verzerrungen [10]. Diese sogenannten Denkfehler sind angstfördernd und begünstigen problematische Verhaltensweisen. Die Gedanken sind in diesem Fall negativ getönt und der Gedankenfokus ist hoffnungsloser sowie pessimistischer. Dieses verzerrte Denken entspricht nicht der Wahrheit oder der Wirklichkeit, sondern ist überzogen negativ. Typische Denkfehler beinhalten Selbstzweifel, Abwertungen der eigenen Person und eine negative Sicht der Lebenssituation sowie der Zukunft. Sie laufen meist automatisch ab, da sie über viele Jahre erlernt wurden und erscheinen einem daher als wahr.

mittleren Erregungsniveau am besten. Es wäre also nicht sinnvoll, in der Prüfungsvorbereitung und/oder einer Leistungssituation auf völlige Entspannung hinzuarbeiten.

\section{Dauerhafte Senkung der Grundanspannung}

Es gibt verschiedene Möglichkeiten, für Entspannung zu sorgen. Am besten ist es, verschiedene Techniken auszuprobieren und die für sich passendste auszuwählen. Bei allen Methoden gilt allerdings, dass eine entspannungsfördernde Wirkung nur bei regelmäßiger Übung erzielt werden kann. Es ist also hilfreich, die Übungen in den täglichen Lernplan beziehungsweise in den Alltag mit einzuplanen.

Beispiele für Entspannungstechniken sind Autogenes Training, Progressive Muskelentspannung nach Jacobson, Meditation und Atemübungen. Sportvereine, Fitnessstudios und Volkshochschulen bieten Kurse dazu an und im Internet finden sich ebenso Anleitungen dazu.

\section{Strategien auf gedanklicher Ebene}

„Ich schaffe es nie das alles zu lernen. Ich werde versagen. Ich bekomme sicherlich in der Prüfung kein Wort heraus.“ - das sind typische Gedanken, die Prüfungsängste auslösen sowie verstärken können. 
\ab. 1 Beispiel eines Gedankenprotokolls eines Studierenden.

\section{Situation}

Aktuelle Ereignisse, die zu unangenehmen Gefühlen führen.

- Es ist 2 Tage vor meiner Prüfung.

- Ich sitze zu Hause am Schreibtisch und lerne.

\section{Automatische Gedanken}

Gedanken, die in dieser Situation aktiviert werden.

- Ich falle bestimmt durch.

- Wahrscheinlich fällt mir nichts von dem Gelernten ein und ich versage auf der ganzen Linie.

\section{Konsequenzen}

Gefühle und Verhalten

- Angst

- Ich bleibe am Schreibtisch sitzen und lerne bis lange in die Nacht, obwohl ich mich nicht mehr konzentrieren kann.

- Tab. 2 Beispiel eines Studierenden.

\section{Alternative, angemessenere Gedanken}

Kann ich die Situation auch anders betrachten? Welche Gedanken sind für mich hilfreicher und realistischer?

- Ich kann zum jetzigen Zeitpunkt nicht wissen, ob ich durchfalle.

- Ich lerne so gut es geht und versuche, entspannt in die Prüfung zu gehen.

- Wenn mir während der Prüfung etwas nicht einfällt, ist das nicht schlimm, dann mache ich mit einer anderen Aufgabe weiter und versuche es später nochmal.

- Ich kann es schaffen, ich habe auch schon andere Prüfungen gemeistert.

\section{Ergebnis}

Gefühle und Verhalten

- Nervosität

- Ich lerne nicht länger als 3 Stunden und lese dann noch ein Kapitel in meinem Buch, zum Entspannen und als Belohnung für die Lerneinheit.
Die Bewertung der eigenen Leistung als nicht ausreichend oder die Angst vor Versagen lassen eine objektiv bewältigbare Situation zu einer bedrohlichen werden. Solche Gedanken unterliegen aber meist sogenannten Denkfehlern und sind katastrophisierend und unrealistisch.

Die nachfolgende Auflistung zeigt einige typische Gedankenfehler und Verzerrungen, die auftreten können:

- Alles-oder-nichts-Denken: zum Beispiel „Wenn ich in der Prüfung eine schlechte Note bekomme, brauche ich gar nicht erst weiterzumachen.“

" Katastrophisieren: zum Beispiel „Ich werde vor lauter Aufregung kein Wort rausbringen.“

- Gedankenlesen: zum Beispiel „Der Prüfer guckt so streng. Meine Antworten sind falsch.“

- Emotionale Beweisführung: zum Beispiel „Wenn meine Überzeugung, durchzufallen, nicht stimmen würde, dann würde ich mich nicht so fühlen.“

Hilfreich ist hier, möglichst angemessene und realistische Gedanken hinsichtlich einer bevorstehenden Prüfung zu entwickeln [11]. Angstauslösende und -aufrechterhaltende Gedanken sollen verringert sowie angstreduzierende Gedanken gesteigert werden. In der kognitiven Verhaltenstherapie werden dysfunktionale oder hinderliche Gedanken in 3 Schritten bearbeitet:

- wahrnehmen/identifizieren,

- hinterfragen,

- umformulieren.

\section{EXKURS: UMGANG MIT GRÜBELN}

Grübeln führt im Gegensatz zum Nachdenken zu keiner Lösung, sondern die Gedanken und Sorgen drehen sich im Kreis und gehen mit einer negativen Stimmung einher. Im Rahmen einer Prüfungsangst betrifft das häufig Gedanken zur Prüfungsvorbereitung und zum vermuteten Ergebnis der Prüfung (zum Beispiel „Ich schaffe das nie, alles zu lernen.“). Um Grübelprozesse zu unterbrechen, bietet sich eine Technik aus der kognitiven Verhaltenstherapie an: der „Gedankenstopp“. Die Betroffenen stellen sich ein Stoppschild aus dem Straßenverkehr vor, sobald sie merken, dass sie in Grübelschleifen geraten. Dadurch entsteht eine plötzliche Unterbrechung, die es ermöglicht, sich kurz von den angstverstärkenden Gedanken abzulenken und anderen Gedanken und Tätigkeiten zu widmen.

\section{Strategien auf der Gefühlsebene}

Ein entscheidender Faktor hierbei ist die Akzeptanz unangenehmer Gefühle. Angst und andere damit einhergehende Gefühle gehören zum Leben und Lernen dazu. AuBerdem bestehen sie nicht dauerhaft, sondern vergehen wieder und können sich verändern. Achtsamkeit, also die gezielte und nicht wertende Wahrnehmung des Augenblicks, stellt eine Strategie im Umgang mit Ängsten dar [13]. Ziel dabei ist es, Gefühle zuzulassen, ohne diese di- 
PRAXISTIPP: BEWÄLTIGUNGSSKRIPTE

Eine weitere Technik im Umgang mit angstauslösenden Gedanken ist das „mentale Training“ [9]. Vor allem Sportler und Musiker wenden diese Methode an, um neue Denk- und Verhaltensmuster einzuüben. Dabei werden zum Beispiel bestimmte Bewegungsabläufe sehr konkret in der Vorstellung durchgegangen. Ein positives Bild einer bevorstehenden Situation löst eine gute Stimmung aus und setzt Energie frei, die zum Lernen und Vorbereiten genutzt werden kann. Dazu stellt sich der Betroffene zum Beispiel den Prüfungstag oder die Prüfung selbst sehr detailliert und mit einem positiven Verlauf und Ende vor. Es ist wichtig, das Bewältigungsskript regelmäßig, am besten täglich, zu lesen.

\section{Beispiel eines Bewältigungsskripts eines Studierenden vor einer schriftlichen Prüfung} Am Tag meiner Klausur stehe ich rechtzeitig auf und mache mir ein gesundes Frühstück. Dabei höre ich meine Lieblingsmusik. Die nötigen Unterlagen habe ich schon am Vorabend gepackt. Ich fahre etwas früher los und gehe schon mal zu dem Raum, in dem die Prüfung stattfinden wird.

Wenn die Blätter ausgeteilt werden, atme ich noch einmal tief ein und aus und beginne dann mit einer ersten Durchsicht der Aufgaben. Ich fange mit der Aufgabe an, zu der mir gleich etwas einfällt und arbeite mich langsam und konzentriert vor. Wenn ich etwas nicht auf Anhieb weiß, bleibe ich ruhig, denke nach oder verschiebe die Aufgabe auf später.

Drängen sich zwischendurch Angstgedanken auf, dann nehme ich sie kurz wahr und konzentriere mich wieder auf das vor mir liegende Aufgabenblatt. Wenn ich alles bearbeitet habe und mir noch Zeit bleibt, lese ich mir die Fragen und meine Antworten noch einmal durch.

Nach der Prüfung treffe ich mich mit meiner besten Freundin auf einen Kaffee in der Stadt.

rekt verändern zu müssen. Durch gezieltes Training der Achtsamkeit lässt sich die Toleranz und damit auch Akzeptanz gegenüber negativen Gefühlen verbessern. Mit etwas Übung hilft Achtsamkeit, Ängste auch direkt positiv zu beeinflussen. Mittel- und langfristig haben die gedanklichen und verhaltensbezogenen Bewältigungsstrategien natürlich ebenfalls einen positiven Effekt auf die Prüfungsangst.

\section{Strategien auf der Verhaltensebene}

Zunächst ist es wichtig, auf die körperlichen Grundbedürfnisse zu achten. In der Prüfungsvorbereitung ist es unerlässlich, genügend Schlaf zu haben, sich regelmäßig, ausreichend und ausgewogen zu ernähren, genügend zu trinken, sich zu bewegen, Sauerstoffzufuhr zu gewährleisten und auf den Konsum von Genuss- und Rauschmitteln mög-
PRAXISTIPP: ZEITIMANAGEMENT

Zeitmanagementtechniken, wie Projekt- oder Lernpläne, helfen, den Überblick zu behalten. Je nachdem wie umfangreich der Lernstoff ist beziehungsweise wie viele Prüfungen abgelegt werden müssen, können Wochen- oder sogar Monatspläne sinnvoll sein. Dazu werden alle festen, nicht verschiebbaren Termine (zum Beispiel Arbeit, Arzttermine) notiert. Als nächstes werden die freien Zeitfenster verplant. Es gilt, dabei auf die persönliche Leistungskurve zu achten. Lernphasen sollten in leistungsstarken, alltäglichen Aufgaben (zum Beispiel Einkaufen, Haushalt et cetera) und in weniger leistungsstarken Phasen terminiert werden. Auch Erholungsphasen und Freizeitaktivitäten sollten eingeplant werden, das motiviert und gibt neue Energie zum Weiterlernen. Um von Unvorhergesehenem nicht aus der Bahn geworfen zu werden, sollten Pufferzeiten oder, wenn möglich, ganze Puffertage miteinbezogen werden.

Bei Schwierigkeiten, einen Plan zu erstellen, können in einem ersten Schritt eine Woche lang alle Aktivitäten beobachtet und protokolliert werden. Dieser IST-Plan gibt einen Eindruck davon, mit welchen Tätigkeiten aktuell wie viel Zeit verbracht wird. Darauf aufbauend kann dann ein SOLL-Lernplan erstellt werden.

\section{PRAXISTIPPS: LERNTECHNIKEN}

Bei der Bewältigung großer Stoffmengen oder beim Verstehen schwieriger Sachverhalte können Lernund Gedächtnistechniken helfen, zum Beispiel die Methode der Orte, Mindmaps, SQR3-Technik. Die Vorbereitung auf die Prüfung sollte an die Art der Prüfung angepasst werden. Handelt es sich um eine mündliche Prüfung, dann sollte der Lernende den Lernstoff laut aufsagen oder sich abfragen lassen. Um sich auf eine schriftliche Prüfung vorzubereiten, kann das Gelernte anhand von alten Klausuren beziehungsweise früheren Prüfungsfragen aus dem Gedächtnis niedergeschrieben werden. Prüfungen am Patienten sollten praktisch geübt werden. Dasselbe gilt für Multiple-Choice-Klausuren. Auf diese Art der Wissensabfrage bereitet Üben mit Beispielaufgaben am besten vor.

lichst zu verzichten beziehungsweise diesen zu reduzieren. So wird die Grundlage für eine gute Prüfungsvorbereitung geschaffen.

Um den Teufelskreis der Angst zu durchbrechen, muss Vermeidungsverhalten abgebaut werden und die Betroffenen müssen sich gefürchteten Situationen stellen. Im 


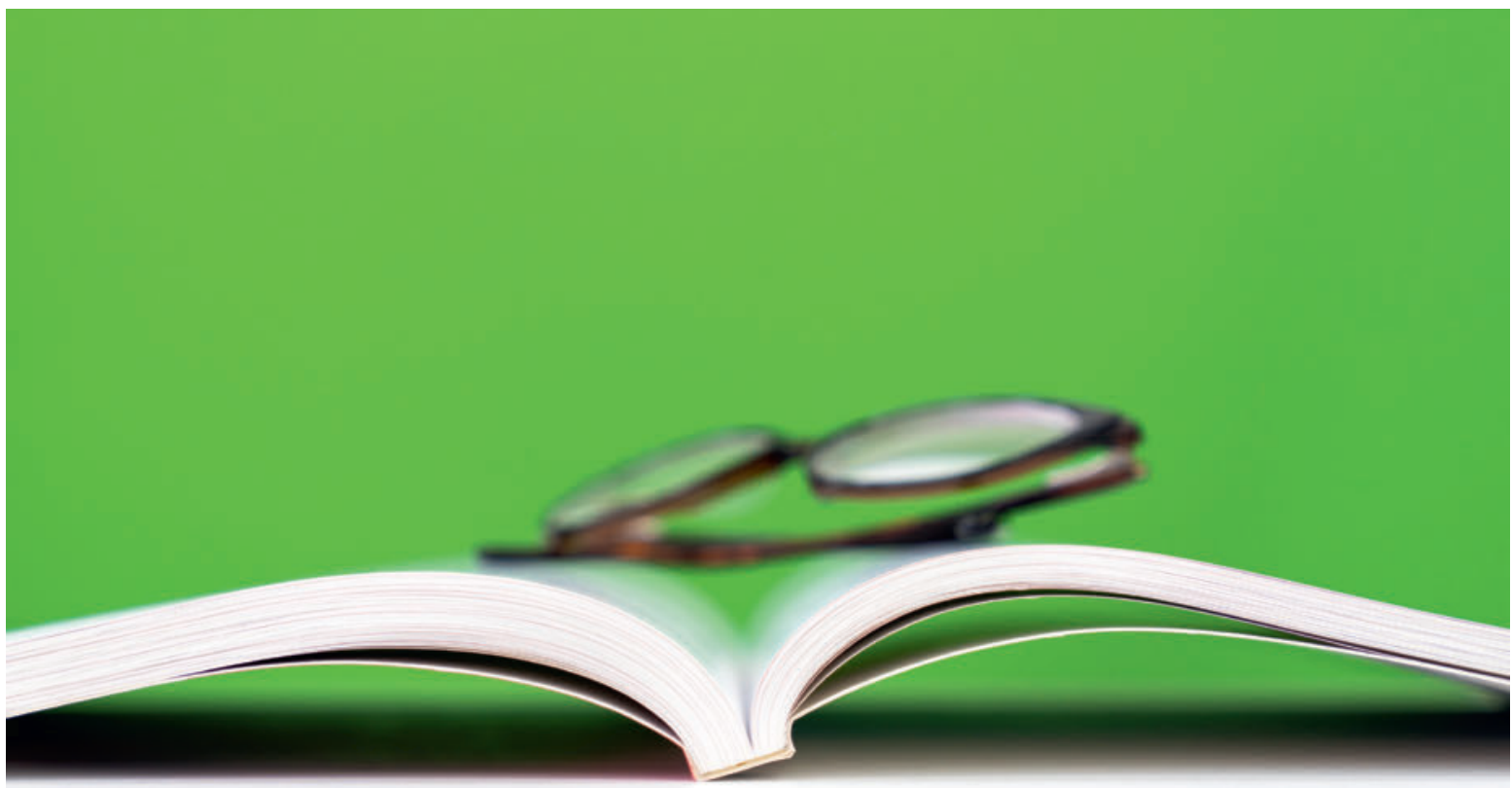

Zu schwieriger Lernstoff, Zeitdruck oder ein Gefühl der Überforderung kann zu Prüfungsängsten führen. (Quelle: Kirsten Oborny/ Thieme Gruppe)

Fall der Prüfungsangst bedeutet dies, sich (rechtzeitig) auf die Prüfung vorzubereiten sowie die Prüfung anzutreten [14].

Wie in anderen stressigen Lebensphasen auch, ist es hilfreich, sich in der Prüfungsphase soziale Unterstützung bei Freunden und Familie zu suchen. Das Erzählen von Sorgen und Ängsten entlastet. Des Weiteren kann das soziale Umfeld auch praktische Unterstützung liefern, zum Beispiel Lernstoff abfragen oder andere Aufgaben abnehmen.

\section{PRAXISTIPPS: LERNGRUPPEN UND PRÜFUNGSSIMULATION}

Ein weiterer Tipp ist es, Lerngruppen zu bilden. Zum einen erhöht sich die Verpflichtung und zum anderen erhöht sich dadurch auch die Motivation. Außerdem können sich die Lernenden gegenseitig Fragen beantworten und sich in Prüfungssimulationen abfragen. So können schwierige Prüfungssituationen vorbereitet und geübt werden, indem der „Prüfer“ verschiedene Rollen einnimmt: mal streng, mal nett.

\section{Besondere Situationen in Prüfungen}

\section{Umgang mit Blackouts}

Ein Blackout geht mit einer starken Aufregung einher. Zunächst gilt es, den Moment größter Angst auszuhalten.
Die Wirkung und Reaktion auf einen Blackout hängen auch von der Bewertung der Situation ab. Gedanken wie „Das ist eine Katastrophe, jetzt falle ich durch. "verstärken die Angst noch zusätzlich. Eine hilfreiche Einstellung ist, dass ein Blackout keine Katastrophe darstellt und ein Umgang damit möglich ist [6].

\section{PRAXISTIPPS: BLACKOUT}

Der 1.Schritt ist, die Tatsache zu akzeptieren, dass ein Blackout in einer Prüfung auftreten kann. Der Betroffene sollte sich zunächst auf seinen Atem konzentrieren und 1-mal tief ein- und ausatmen, um seine Ruhe zurückzugewinnen. Im Vorhinein kann überlegt werden, was der Prüferin oder dem Prüfer im Falle eines Blackouts gesagt werden könnte (zum Beispiel um Verständnis, eine Hilfestellung oder eine kurze Pause bitten).

\section{Umgang mit Misserfolgen}

Falls eine Prüfung nicht so läuft wie erhofft (schlechte Note oder nicht bestanden), dann ist das ärgerlich, aber das kann passieren. Entscheidend ist, wie der Betroffene mit diesem Ereignis umgeht. Die Gedanken und Bewertungen sollten nicht selbstabwertend sein. Die konkreten Umstände, wie es dazu gekommen sein könnte, sollten möglichst objektiv analysiert werden. Denn daraus lassen sich in einem nächsten Schritt Konsequenzen für die nächste Prüfungsphase oder Prüfung ziehen. 


\section{FAZIT}

Viele Menschen sind im Laufe ihres Lebens von Prüfungsängsten betroffen. Diese Angst reicht von leichter Nervosität bis hin zu ausgeprägter Panik. Prüfungen stellen eine besondere Leistungsanforderung dar und sind häufig mit dem Beginn eines neuen Ausbildungs- oder sogar Lebensabschnitts verbunden. Somit ist es menschlich, aufgeregt zu sein. Wird die Angst so stark, dass sie einen daran hindert, seine beruflichen Ziele zu verfolgen, ist es wichtig, sich professionelle Hilfe zu holen (beispielsweise bei einem Psychologischen Psychotherapeuten). Es gibt verschiedene Techniken, die bei der Bewältigung von Prüfungsängsten helfen. Die Herausforderung zu akzeptieren und gelernte Strategien regelmäßig zu üben, verhilft zu einem erfolgreichen Prüfungsergebnis.

\section{Autorinnen}

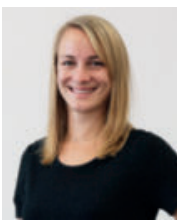

\section{Caroline Lutz-Kopp}

Psychologische Psychotherapeutin (VT), seit 2010 in der Psychotherapeutischen Beratungsstelle der JGU Mainz (Einzelberatung und Kurse); zudem Leitung des Projekts „Erstellung eines Online-Programms zur Stärkung der psychischen Gesundheit Studierender (me@JGU)“

E-Mail: caroline.lutz-kopp@uni-mainz.de

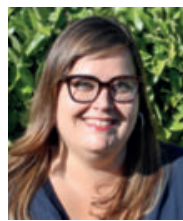

\section{Dr. Maria Gropalis}

Psychologische Psychotherapeutin (Schwerpunkt VT), seit 2018 Leiterin der Psychotherapeutischen Beratungsstelle der JGU Mainz; zuvor 10 Jahre in der Ausbildung von Psychologischen Psychotherapeuten an der JGU Mainz tätig, in diesem Zusammenhang außerdem Referentin und Supervisorin
[1] Dilling H, Freyberger H], Hrsg. Taschenführer zur ICD10-Klassifikation psychischer Störungen: Mit Glossar und Diagnostischen Kriterien sowie Referenztabellen ICD-10 vs. ICD-9 und ICD-10 vs. DSM-IV-TR. Göttingen: Hogrefe; 2019

[2] Shapiro AL. Test anxiety among nursing students: A systematic review. Teaching and Learning in Nursing 2014; 9: 193-202

[3] Quek TT, Tam WW, Tran BX et al. The Global Prevalence of Anxiety Among Medical Students: A Meta-Analysis. International Journal of Environmental Research and Public Health 2019; 16: 2735. doi:10.3390/ijerph16152735

[4] Psychotherapeutische Beratungsstelle der Johannes Gutenberg-Universität Mainz Jahresbericht 2018. Im Internet: von: https://www.pbs.uni-mainz.de/ (files/2019/07/Jahresbericht_PBS_2018.pdf); Stand: 27.02.2020

[5] Fehm L, Fydrich T. Prüfungsangst. Göttingen: Hogrefe; 2011

[6] Warnecke I. Prüfungsangst bewältigen: Ein Trainingsprogramm in sieben Schritten. Stark fürs Studium, Band 4857. Stuttgart: UTB; 2018

[7] Ergene T. Effective Interventions on test anxiety reduction A meta-analysis. School Psychology International 2003; 24 (3): 313-328

[8] Hofmann SG, Asnaani A, Vonk IJ] et al. The efficacy of cognitive behavioral therapy: a review of meta-analyses. Cognitive Therapy and Research 2012; 36 (5): 427-440. doi:10.1007| s10608-012-9476-1

[9] Wolf D, Merkle R. So überwinden Sie Prüfungsängste. Psychologische Strategien zur optimalen Vorbereitung und Bewältigung von Prüfungen. Mannheim: PAL; 2017

[10] Beck JS. Praxis der Kognitiven Verhaltenstherapie. Weinheim, Basel: Beltz; 2013

[11] Wilken B. Methoden der Kognitiven Umstrukturierung. Ein Leitfaden für die psychotherapeutische Praxis. Stuttgart: Kohlhammer; 2018

[12] Beck AT. Praxis der Kognitiven Therapie. Weinheim: Beltz; 1999

[13] Kabat-Zinn J. Im Alltag Ruhe finden. Frankfurt a. M.: Fischer; 2009

[14] Fehm L, Fydrich T. Ratgeber Prüfungsangst. Informationen für Betroffene und Angehörige. Göttingen: Hogrefe; 2013

Bibliografie

DOI https://doi.org/10.1055/a-1124-1587

Psych. Pflege Heute 2020; 26: 138-145

(c) Georg Thieme Verlag KG Stuttgart · New York

ISSN 0949-1619 